\title{
Sample-size properties of a case-control association analysis of multistage SNP studies for identifying disease susceptibility genes
}

\author{
Nobutaka Kitamura $\cdot$ Kouhei Akazawa • \\ Shin-ichi Toyabe $\cdot$ Akinori Miyashita \\ Ryozo Kuwano $\cdot$ Junichiro Nakamura
}

Received: 5 November 2007/ Accepted: 24 January 2008/Published online: 21 February 2008

(C) The Japan Society of Human Genetics and Springer 2008

\begin{abstract}
A two-stage association study is the most commonly used method to efficiently identify disease susceptibility genes. However, some recent single nucleotide polymorphism (SNP) studies recently utilized threestage designs. The purpose of this study was to investigate the practical properties of statistical powers and positive predictive values (PPVs) of replication-based analysis (RBA) and the joint analysis (JA) in multistage designs. For this purpose, a program for multistage designs was developed to calculate these performance indicators under various conditions of the number of samples, alleles of candidates, alleles remaining in the final stage, and genotypings. The results showed that the powers and PPVs of RBA and JA in three-stage designs were higher than those in two-stage designs in the range of a smaller proportion of sample size than 0.5 at the first stage. This tendency was more remarkable in JA. In conclusion, researchers who perform SNP studies for identifying disease susceptibility genes need to take account of three-stage case-control association studies, corresponding to study conditions such as the total numbers of samples, alleles, and genotypings. Furthermore, the program developed in this study is useful for estimating powers and PPVs in planning multistage association studies.
\end{abstract}

N. Kitamura · K. Akazawa $(\bowtie) \cdot$ S. Toyabe $\cdot$ J. Nakamura Department of Medical Informatics, Niigata University Medical and Dental Hospital, 1-754 Chuo-ku, Asahimachi-dori, Niigata 951-8520, Japan

e-mail: akazawa@med.niigata-u.ac.jp

A. Miyashita $\cdot$ R. Kuwano

Department of Molecular Genetics, Bioresource Science Branch, Center of Bioresources, Brain Research Institute,

Niigata University, Niigata 951-8585, Japan
Keywords Case-control association analysis - Multistage design $\cdot$ Joint analysis $\cdot$ Replication-based analysis

\section{Introduction}

Genome-wide association (GWA) case-control studies are carried out to identify susceptibility genes of common diseases by using single nucleotide polymorphisms (SNPs) and/or microsatellite markers (Tamiya et al. 2005; Samani et al. 2007; The Wellcome Trust Case Control Consortium 2007). However, GWA case-control studies require genotyping of hundreds of thousands of markers in many subjects, and they are expensive at current genotyping costs, even though chip-based genotyping products have recently decreased these costs (Service et al. 2003; Skol et al. 2007). Therefore, efficient study designs are needed for GWA casecontrol studies (Hirschhorn and Daly 2005; Cohen 2006).

Recently, some multistage GWA case-control study designs have been proposed as powerful and cost-effective study designs. For example, two-stage designs genotype all markers for the predetermined portion of the overall sample in the first stage and once again genotype only the significant markers selected in first stage for the remaining sample in the second stage. At the end of the second stage, the overall evidence for association is evaluated by replication-based analysis (RBA) (Satagopan et al. 2002) or joint analysis (JA) (Skol et al. 2006, 2007). Thus, it is expected that two-stage designs will exclude almost all markers that show little evidence of association in the first stage. There have been some theoretical studies and simulations regarding the statistical power, positive predictive value (PPV), and cost reduction of two-stage designs. However, because two-stage designs divide the overall samples into two subgroups, the power of each statistical test will decrease, resulting in an 
increase in the probability of defeating true disease susceptibility genes. Therefore, how to maintain the power while decreasing the number of genotypings is the most important consideration for multistage design.

On the other hand, some genetic studies have adopted three-stage designs (Prentice and Qi 2006; Kathiresan et al. 2007). When the number of SNPs analyzed is very large (Moore and Ritchie 2004), a three-stage design may be realistic to efficiently identify SNPs associated with some diseases from the point of view of maintaining higher powers and reducing the number of genotypings in conjunction with study costs. Prentice and Qi (2006) performed a high-dimensional SNP-based association study with 250,000 SNPs and described the outline of the three-stage study of the Women's Health Initiative Project to identify genetic risk factors for coronary heart disease, stroke, and breast cancer and other genetic factors related to the effect of combined postmenopausal hormone therapy on any of these three diseases. They conducted a simulation study for the design and reported that their adequate three-stage design is expected to have slightly lower power while greatly decreasing cost compared with a one-stage design. Moreover, Kathiresan et al. (2007) conducted genome-wide analyses to map genetic loci related to three phenotypes of heritable blood lipid levels in the Framingham Heart Study. They pursued a three-stage design by using three independent sample sets as replication samples to maximize statistical powers and minimize the number of genotypings. In their strategy, a large number of markers (100 K Gene Chip) were screened genome-wide at first stage, and the selected moderate number of markers (287 SNPs) were screened at the second stage. Then, the more promising small number of markers (40 SNPs) were retested at the third stage. However, there have been few other studies on the statistical properties of the powers and PPVs of a three-stage design.

In this paper, we show the sample-size properties of RBA and JA regarding statistical powers, PPVs, and the number of genotypings by comparing three-stage designs with conventional two-stage designs. The parameters for calculating the performance indicators in this study include the proportion of sample sizes allocated to each stage, the number of candidate SNPs, and the significance level at each stage.

\section{Materials and methods}

Statistical tests for detecting risk SNPs

We show the outline of RBA and JA to detect SNPs that have significant differences in the frequencies of risk alleles between the case and control groups in $n$-stage association designs, where $n$ is $\geq 2$. In addition, the method for calculating the powers and PPVs is described.

Let $N$ be the sums of samples in cases and controls in a case-control study, and let $\pi_{s, k}$ (where $s$ is an abbreviation of samples and $k=1, \ldots, n$ ) be a proportion of the sample size at the $k$ th stage. Let $p^{\prime}$ and $p$ be the disease allele genotype rates of SNPs in the cases and controls in a population, respectively, and let $\hat{p}^{\prime}$ and $p^{\prime}$ be the estimated disease allele genotype rates in the cases and controls, respectively.

In the $k$-stage design, a test statistic $z_{k}$ is calculated using the sample of the $k$ th stage and is compared with critical value $C_{k}$ determined by the following procedure. Under the null hypothesis of no association $\left(p^{\prime}=p\right)$, the statistic

$z_{k}=\frac{\hat{p}^{\prime}-\hat{p}}{\sqrt{\left[\hat{p}^{\prime}\left(1-\hat{p}^{\prime}\right)+\hat{p}(1-\hat{p})\right] / 2 N \pi_{s, k}}}$

approximately follows a standard normal distribution. Similarly, under the alternative hypothesis, a test statistic $z_{k}$ approximately follows a normal distribution with mean

$\mu_{k}=\frac{p^{\prime}-p}{\sqrt{\left(p^{\prime}\left(1-p^{\prime}\right)+p(1-p)\right) / 2 N \pi_{s, k}}}$

and variance 1 .

Replication-based analysis (RBA) in $n$-stage design

Let $\pi_{m, k}$ (where $m$ is an abbreviation of markers and $k=1$, $\ldots, n)$ be a proportion of alleles to be selected at the $k$ th stage, that is,

$\pi_{m, k}=P\left\{z_{k}|| z_{k} \mid>C_{k}\right\} \quad(k=1, \cdots, n-1)$,

where $P\left\{z_{k}\right\}$ represents a probability of $z_{k}$. $\pi_{m, k}$ can be determined in consideration of multiple comparison. Let $\Phi[x]$ be the cumulative distribution function for a standard normal distribution evaluated at $x$ and $\Phi^{-1}[\cdot]$ the inverse function of $\Phi[x]$. Then, $C_{k}$ can be calculated for two-tailed tests as follows:

$C_{k}=\Phi^{-1}\left[1-\pi_{m, k} / 2\right]$.

Furthermore, let $\alpha_{\text {genome }}$ be the genome-wide false positive rate, $M$ the number of candidate alleles, and $C_{n}$ the critical value for the $n$th stage. Under the null hypothesis, $C_{n}$ can be obtained by finding the value that satisfies the following equation: 


$$
\begin{aligned}
\pi_{m, n} & =P\left\{z_{n}|| z_{n} \mid>C_{n}, \operatorname{sign}\left(z_{1}\right)=\cdots=\operatorname{sign}\left(z_{n}\right)\right\} \\
& =\frac{\alpha_{\text {genome }}}{P\left\{\left(z_{1}, \cdots, z_{n-1}\right)|| z_{1}\left|>C_{1}, \cdots,\right| z_{n-1} \mid>C_{n-1}, \operatorname{sign}\left(z_{1}\right)=\cdots=\operatorname{sign}\left(z_{n-1}\right)\right\} \times M} .
\end{aligned}
$$

Then, we represent the powers, $P_{1}$ and $P_{r, n}$ (where $r$ is an abbreviation of replication), in the first stage and the $n$th stage $(n \geq 2)$ are as follows, respectively:

$P_{1}=1-\Phi\left[C_{1}-\mu_{1}\right]+\Phi\left[-C_{1}-\mu_{1}\right]$

$P_{r, n}=\frac{\prod_{k=1}^{n}\left(1-\Phi\left[C_{k}-\mu_{k}\right]\right)+\prod_{k=1}^{n} \Phi\left[-C_{k}-\mu_{k}\right]}{1-\Phi\left[C_{1}-\mu_{1}\right]+\Phi\left[-C_{1}-\mu_{1}\right]}$.

Therefore, the power of RBA by an $n$-stage design is $P_{1} P_{r, n}$.

Joint analysis (JA) in $n$-stage design

The test statistic $z_{j, n}$ of JA between the first stage from the $n$th stage is represented as the weighted sum of $z_{k}$ $(k=1, \ldots, n)$ as follows:

$z_{j, n}=\sum_{k=1}^{n} \sqrt{\pi_{s, k}} z_{k}$

Conditional on the observed $k$ th stage statistic $z_{k}=a_{k}\left(a_{k}\right.$ : constant, $k=1, \ldots, n-1)$, the test statistic, $z_{j, n}$ of JA, approximately follows a normal distribution with the mean

$\mu_{j, n}=\frac{p^{\prime}-p}{\sqrt{\left[p^{\prime}\left(1-p^{\prime}\right)+p(1-p)\right] /(2 N)}}+\sum_{k=1}^{n-1} \sqrt{\pi_{s, k}}\left(a_{k}-\mu_{k}\right)$

and variance $1-\sum_{k=1}^{n-1} \pi_{s, k}$. To calculate the critical value of JA, $C_{j, n}$, and the power, we think the following equation of $n$-multiple integrals:
JA in an $n$-stage design can be calculated as $P_{j, n}$ with the critical value $C_{j, n}$ under the alternative hypothesis.

Next, we show the method for calculating PPV. Let $m$ be the number of true alleles that truly associate with the disease in $M$ candidate alleles. Then, the number of true positive alleles $\left(\mathrm{tp}_{k}\right)$ and false positive alleles $\left(\mathrm{fp}_{k}\right)$ remaining at the $k$ th stage, respectively, are given as follows.

The number of true positive alleles at the $k$ th stage is given as

$\operatorname{tp}_{k}=\left\{\begin{array}{cc}m \times P_{1} \times P_{r, k} & (\text { in the case of RBA) } \\ m \times P_{j, k} & \text { (in the case of JA) }\end{array}\right.$

and the number of false positive alleles at the $k$ th stage is given as

$\mathrm{fp}_{k}=(M-m) \times \prod_{k=1}^{n} \pi_{m, k}$.

Therefore, $\mathrm{PPV}_{k}$ at the $k$ th stage is calculated as $\mathrm{PPV}_{k}=\mathrm{tp}_{k} /\left(\mathrm{tp}_{k}+\mathrm{fp}_{k}\right)$.

\section{Calculation of powers and PPVs}

The programs for calculating the powers and PPVs of RBA and JA were created using $\mathrm{R}$ language.

To confirm the precision of these programs, the results of JA in the two-stage design were compared with those reported by Skol et al. (2006). As a result of the compar-

$$
\begin{aligned}
P_{j, n}= & \int_{-\infty}^{-c(n-1)} \cdots \int_{-\infty}^{-c_{2}} \int_{-\infty}^{-c_{1}}\left\{P\left[z_{j, n}>C_{j, n} \mid \bigcap_{k=1}^{n-1}\left\{z_{k} \mid z_{k}=x_{k}\right\}\right]+P\left[z_{j, n}<-C_{j, n} \mid \bigcap_{k=1}^{n-1}\left\{z_{k} \mid z_{k}=x_{k}\right\}\right]\right\} \\
& \times f\left(x_{1}|| z_{1} \mid>C_{1}\right) \cdots f\left(x_{n-1}|| z_{n-1} \mid>C_{n-1}\right) \mathrm{d} x_{1} \cdots \mathrm{d} x_{n-1} \\
& +\int_{c(n-1)}^{\infty} \cdots \int_{c_{2}}^{\infty} \int_{c_{1}}^{\infty}\left\{P\left[z_{j, n}>C_{j, n} \mid \bigcap_{k=1}^{n-1}\left\{z_{k} \mid z_{k}=x_{k}\right\}\right]+P\left[z_{j, n}<-C_{j, n} \mid \bigcap_{k=1}^{n-1}\left\{z_{k} \mid z_{k}=x_{k}\right\}\right]\right\} \\
& \times f\left(x_{1}|| z_{1} \mid>C_{1}\right) \cdots f\left(x_{n-1}|| z_{n-1} \mid>C_{n-1}\right) \mathrm{d} x_{1} \cdots \mathrm{d} x_{n-1} .
\end{aligned}
$$

$C_{j, n}$ can be calculated by setting $P_{j, n}=$ $\alpha_{\text {genome }} /\left(M \prod_{k=1}^{n-1} \pi_{m, k}\right)$ under the null hypothesis and solving it by an iterative method. However, the power of ison, different values were found following the fourth decimal place, but these values were rounded off to three decimal places to be corresponding values between the current results and those of Skol et al. 
We assume the following conditions for calculating the powers and PPVs in three-stage designs. The powers and PPVs for one-stage and two-stage designs can be calculated by the methods described by Skol et al. (2006).

\section{Simulation 1}

Assume that the number of cases is 1,000 , the number of controls is $1,000, M=500,000, \alpha_{\text {genome }}=0.05$, and odds ratio $(\mathrm{OR})=1.5$, which is calculated by prevalence $=0.1$, disease allele frequency $=0.4$, and genotype relative risk $=1.61$ in Skol's work (Skol et al. 2006), and the genetic model is set to be an allelic model. The assignment of subjects to each stage was performed in two ways. One way was that $\pi_{s, 1}=0.1-0.9$ as the first stage and the remaining subjects were equally divided to the second and third stages. The other way was that the subjects in the first and second stages were set to be $\pi_{s, 1}=\pi_{s, 2}$ and the remaining subjects were assigned to the third stage. These two procedures are designated as "assignment 1" and "assignment 2." In addition, $\pi_{m, 1}$ and $\pi_{m, 2}$ were set to 0.01 and 0.01 , respectively, to adjust the product of them to 0.0001 .

\section{Simulation 2}

The powers of RBA and JA in a two-stage design were compared with those in a three-stage design under the conditions of $0.1 \leqq \pi_{s, 1} s \leqq 0.9$ and assignment 1 . Furthermore, we assume that the two-stage and three-stage designs have the same numbers of alleles remaining at the final stage, that is, $\pi_{m, 1}=0.0001$ in the two-stage design and $\pi_{m, 1} \times \pi_{m, 2}=0.0001$ in the three-stage design. The other conditions are the same as those in simulation 1 .

\section{Simulation 3}

First, the powers and PPVs of RBA and JA were compared among one-stage, two-stage, and three-stage designs under the conditions of $\mathrm{OR}=1.3,1.5,1.7,0.1 \leqq \pi_{s, 1} \leqq 0.9$, and $\pi_{m, 1}=0.0001$ in the two-stage design and $\pi_{m, 1} \times \pi_{m, 2}=$ 0.0001 in the three-stage design. Next, the powers and PPVs were compared under the condition that the twostage and three-stage designs have the same numbers of genotypings $\left(\pi_{m, 1}=0.00505\right.$ in the two-stage design and $\pi_{m, 1} \times \pi_{m, 2}=0.0001$ in the three-stage design), and ORs were set to $1.3,1.5,1.7$, and $\pi_{s, 1} s$ were set in the range between 0.1 and 0.9 in the two-stage and three-stage designs by assignment 1 .

\section{Results}

Powers of RBAs and JA in three-stage designs in changing the ratio of subjects assigned to each stage

Figure $1 \mathrm{a}, \mathrm{b}$ shows the power curves of RBA and JA in a three-stage design for various $\pi_{s, 1}, \pi_{s, 2}$, and $\pi_{s, 3}$, with $\mathrm{OR}=1.5$ and $\pi_{m, 1}=\pi_{m, 2}=0.01$. The power curves of RBA are unimodal (Fig. 1a), and the powers in assignment 1 are less than 0.2 for $\pi_{s, 1} \geqq 0.7$. The values of $\pi_{s, 1}$ that attain the maximal power are 0.3 for both assignment 1 and assignment 2 in Fig. 1a, respectively. The maximum powers are estimated to be about 0.6 in both assignments in Fig. 1a, b.

On the other hand, the power curves for JA are considerably different for assignment 1 and assignment 2 (Fig. 1b). Namely, the power curve of assignment 1 is unimodal with a maximum power of 0.724 at $\pi_{s, 1}=0.4$, and that of assignment 2 is monotonously increasing as $\pi_{s, 1}$ increases. It is important to note that $\pi_{s, 1}=0.325$ on the curve of assignment 2 is equal to $\pi_{s, 1}=0.4$ on the curve of assignment 1 .

The numbers of genotypings of assignment 1 and assignment 2 in the three-stage design were similar, and with increase in $\pi_{s, 1}$, the number of genotypings linearly increased.

Comparisons of the powers of RBA and JA between two- and three-stage designs

Figure 2 shows the power curves of RBA and JA for two- and three-stage designs under the assumptions of $\mathrm{OR}=1.5$ and $\pi_{m, 1}=0.0001$ in the two-stage design and $\pi_{m, 1}=\pi_{m, 2}=0.01$ for assignment 1 in the three-stage design. The powers of JA and RBA in three-stage designs were higher than those in two-stage designs in the range of $\pi_{s, 1} s$ smaller than 0.5. Specifically, the differences in the powers of JA between two- and three-stage designs are remarkable.

The number of genotypings in the two-stage design and in the three-stage design were of similar value and showed a monotone and linear increase with increase in $\pi_{s, 1}$.

Powers and PPVs of RBA and JA in one-, twoand three-stage designs and detailed statistics necessary for deriving (calculating) powers

Table 1 shows the powers, PPVs, and statistics related to the powers in each design with various values of OR and $\pi_{s, 1}$ under the condition of the same number of alleles remaining at the final stage. With increasing OR, both powers and PPVs of RBA and JA increased. In addition, 

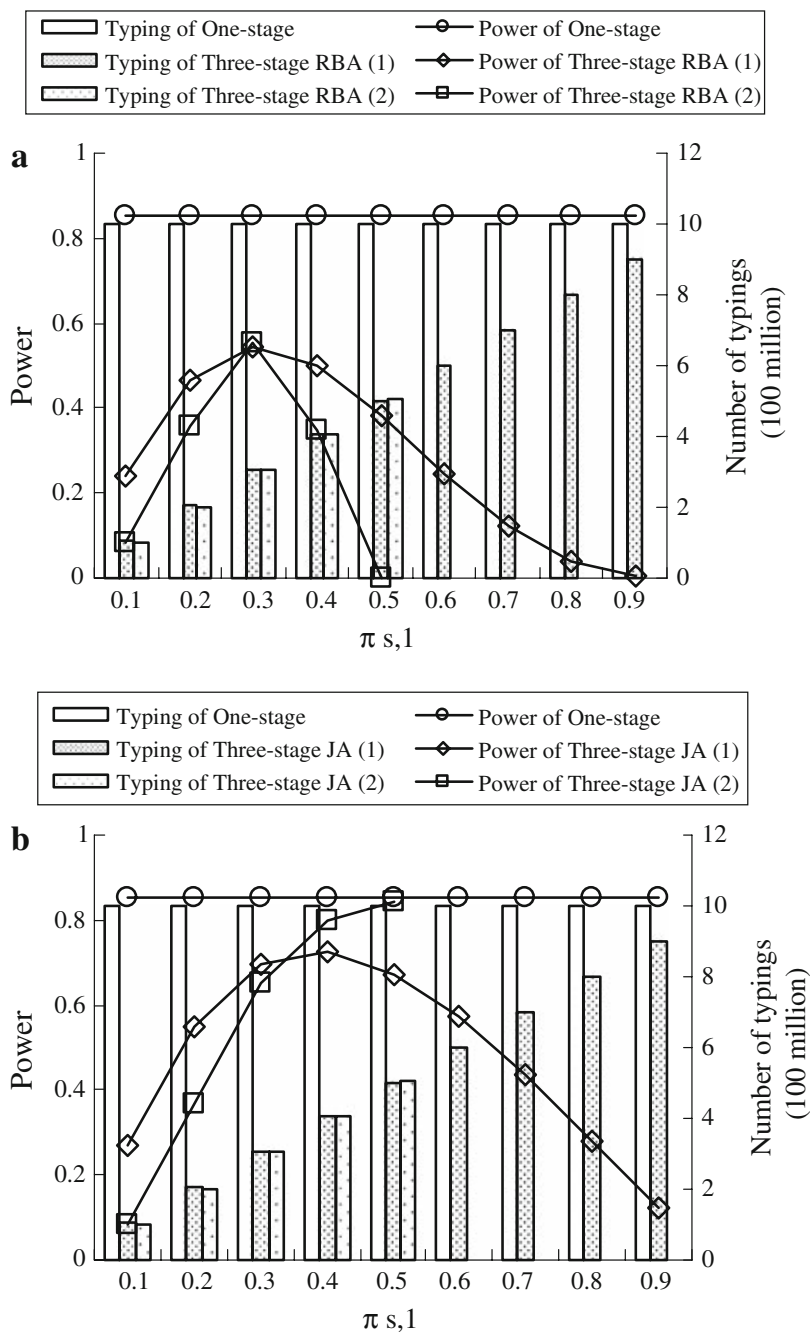

Fig. 1 Changes in the powers of replication-based analysis (RBA) (a) and joint analysis (JA) (b) by a three-stage design with various values of $\pi_{s, 1}, \pi_{s, 2}, \pi_{m, 1}, \pi_{m, 2}$, and odds ratio (OR). The setting condition of the simulation was assumed to be the number of cases $=1,000$, the number of controls $=1,000, M=500,000$, $\alpha_{\text {genome }}=0.05$, allelic model, and OR $=1.5$. Samples were divided in two ways. One way was that $\pi_{s, 1}=0.1-0.9$ as the first stage and the remaining cases were divided equally [assignment 1 in (1)] and the other way was that the first and second stages were set as $\pi_{s, 1}=\pi_{s, 2}$ and the remaining cases were assigned to the third stage [assignment 2 in (2)]. $\pi_{m, 1}$ and $\pi_{m, 2}$ were assumed to be 0.01 and 0.01 , respectively, to adjust the product of them to 0.0001

both powers and PPVs of RBA and JA by the three-stage design were higher than those by the two-stage design in the range of $\pi_{s, 1} s$ smaller than 0.5 . On the other hand, in the range of $\pi_{s, 1} s$ larger than 0.6 , the indicators by the twostage design were higher than those by the three-stage design. Table 2 shows the powers and PPVs by each design with various values of OR and $\pi_{s, 1}$ while assuming that the number of genotypings is fixed. The tendencies in Table 2 were similar to those in Table 1, but the range of higher powers and PPVs of RBA in the three-stage design than

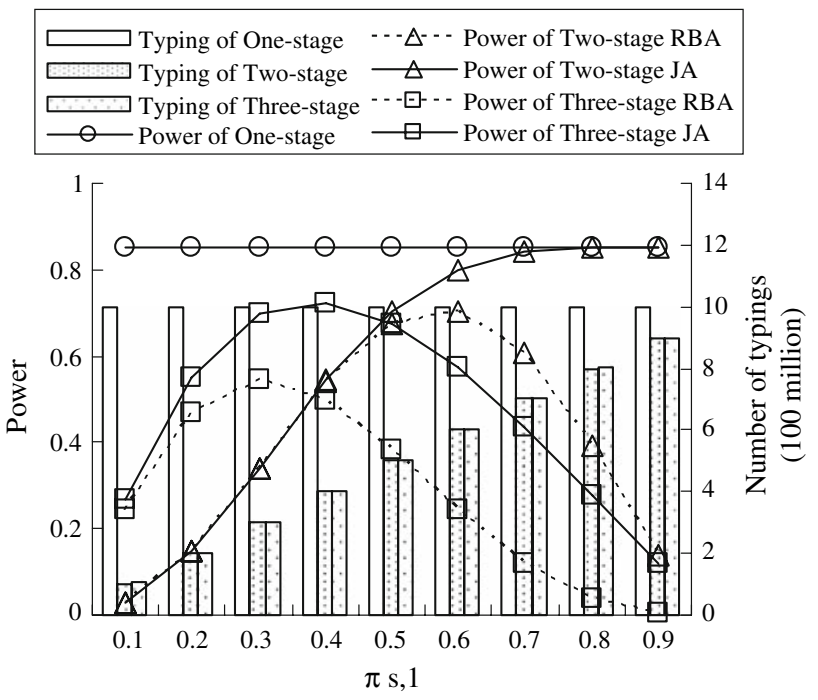

Fig. 2 Changes in the powers of replication-based analysis (RBA) and joint analysis (JA) in two- and three-stage designs with $\pi_{m, 1} \times \pi_{m, 2}$ and $\pi_{s, 1}$. The setting condition of the simulation was assumed to be the number of cases $=1,000$, the number of controls $=1,000, M=500,000, \alpha_{\text {genome }}=0.05$, allelic model, and odds ratio $(\mathrm{OR})=1.5$. The setting of $\pi_{s, 1}$ and $\pi_{s, 2}$ followed Fig. 1 (1) and that of $\pi_{m, 1}$ and $\pi_{m, 2}$ followed Fig. 1

two-stage design became narrow compared with Table 1 . In addition, with increasing OR, the range of higher powers and PPVs of RBA in the three-stage design gradually became larger than that in the two-stage design. In the case of JA, these tendencies were more remarkable.

By definition, $C_{1}, C_{2}$ and $C_{3}$ of RBA in two- and threestage designs remained unchanged, even with increase in $\pi_{s, 1}$ increases. In addition, the powers of the first stage in the three-stage design were always larger than those by the two-stage design, because the significant level of the threestage design was higher than that of the two-stage design. On the other hand, the powers of the final stage in the twostage design were always larger than those in the threestage design. Furthermore, with increasing $\pi_{s, 1}$, the powers of the first stage increased and those of the final stage decreased.

The statistics $C_{j, 2}$ and $C_{j, 3}$ of JA have various behaviors depending on OR and $\pi_{s, 1}$. Generally, $C_{j, 2}$ monotonically increased with increase in $\pi_{s, 1}$, and $C_{j, 3}$ increased with decrease in $\pi_{s, 1}$, reached a maximum at $\pi_{s, 1}=0.5$, and then decreased on the range of higher values. In the range of $0.1-0.5$ of $\pi_{s, 1}, C_{j, 3}$ was higher than $C_{j, 2}$.

\section{Discussion}

It is important to design sophisticated case-control studies for efficient selection of disease susceptibility genes from a large number of genes. The term of "efficient selection" 
Table 1 Performance indicators of two- and three-stage designs calculated by a stage-design program assuming that the number of samples and the number of alleles remaining at the final stage are fixed

\begin{tabular}{|c|c|c|c|c|c|c|c|c|}
\hline \multirow[t]{2}{*}{ OR } & \multirow[t]{2}{*}{ Method } & \multirow[t]{2}{*}{ Design } & \multirow[t]{2}{*}{ Indicator } & \multicolumn{5}{|l|}{$\pi_{s, 1}$} \\
\hline & & & & 0.1 & 0.3 & 0.5 & 0.7 & 0.9 \\
\hline \multirow[t]{23}{*}{1.3} & & One-stage & $\mathrm{C}$ & 5.327 & 5.327 & 5.327 & 5.327 & 5.327 \\
\hline & & & $P_{1}$ & 0.113 & 0.113 & 0.113 & 0.113 & 0.113 \\
\hline & & & PPV & 0.919 & 0.919 & 0.919 & 0.919 & 0.919 \\
\hline & $\mathrm{RBA}$ & Two-stage & $C_{1}$ & 3.891 & 3.891 & 3.891 & 3.891 & 3.891 \\
\hline & & & $P_{1}$ & 0.005 & 0.051 & 0.163 & 0.327 & 0.505 \\
\hline & & & $C_{2}$ & 3.090 & 3.090 & 3.090 & 3.090 & 3.090 \\
\hline & & & $P_{2}$ & 0.792 & 0.638 & 0.428 & 0.201 & 0.037 \\
\hline & & & $P_{r, 2}$ & 0.004 & 0.032 & 0.070 & 0.066 & 0.019 \\
\hline & & & PPV & 0.276 & 0.764 & 0.875 & 0.868 & 0.650 \\
\hline & & Three-stage & $C_{1}$ & 2.576 & 2.576 & 2.576 & 2.576 & 2.576 \\
\hline & & & $P_{1}$ & 0.101 & 0.374 & 0.631 & 0.807 & 0.908 \\
\hline & & & $C_{2}$ & 2.576 & 2.576 & 2.576 & 2.576 & 2.576 \\
\hline & & & $P_{2}$ & 0.573 & 0.444 & 0.302 & 0.163 & 0.049 \\
\hline & & & $C_{3}$ & 3.090 & 3.090 & 3.090 & 3.090 & 3.090 \\
\hline & & & $P_{3}$ & 0.212 & 0.114 & 0.046 & 0.011 & 0.001 \\
\hline & & & $P_{r, 3}$ & 0.022 & 0.042 & 0.029 & 0.009 & 0.001 \\
\hline & & & PPV & 0.811 & 0.895 & 0.852 & 0.639 & 0.118 \\
\hline & JA & Two-stage & $C_{j, 2}$ & 4.244 & 4.877 & 5.177 & 5.306 & 5.327 \\
\hline & & & $P_{j, 2}$ & 0.004 & 0.034 & 0.081 & 0.109 & 0.113 \\
\hline & & & PPV & 0.277 & 0.771 & 0.890 & 0.916 & 0.919 \\
\hline & & Three-stage & $C_{j, 3}$ & 4.927 & 5.186 & 5.204 & 5.092 & 4.815 \\
\hline & & & $P_{j, 3}$ & 0.034 & 0.082 & 0.082 & 0.055 & 0.020 \\
\hline & & & PPV & 0.774 & 0.891 & 0.892 & 0.846 & 0.664 \\
\hline \multirow[t]{23}{*}{1.5} & & One-stage & $\mathrm{C}$ & 5.327 & 5.327 & 5.327 & 5.327 & 5.327 \\
\hline & & & $P_{1}$ & 0.852 & 0.852 & 0.852 & 0.852 & 0.852 \\
\hline & & & PPV & 0.988 & 0.988 & 0.988 & 0.988 & 0.988 \\
\hline & $\mathrm{RBA}$ & Two-stage & $C_{1}$ & 3.891 & 3.891 & 3.891 & 3.891 & 3.891 \\
\hline & & & $P_{1}$ & 0.030 & 0.345 & 0.731 & 0.925 & 0.984 \\
\hline & & & $C_{2}$ & 3.090 & 3.090 & 3.090 & 3.090 & 3.090 \\
\hline & & & $P_{2}$ & 0.998 & 0.988 & 0.922 & 0.656 & 0.141 \\
\hline & & & $P_{r, 2}$ & 0.030 & 0.340 & 0.674 & 0.607 & 0.139 \\
\hline & & & PPV & 0.752 & 0.971 & 0.985 & 0.984 & 0.933 \\
\hline & & Three-stage & $C_{1}$ & 2.576 & 2.576 & 2.576 & 2.576 & 2.576 \\
\hline & & & $P_{1}$ & 0.288 & 0.820 & 0.973 & 0.997 & 1.000 \\
\hline & & & $C_{2}$ & 2.576 & 2.576 & 2.576 & 2.576 & 2.576 \\
\hline & & & $P_{2}$ & 0.955 & 0.884 & 0.729 & 0.457 & 0.125 \\
\hline & & & $C_{3}$ & 3.090 & 3.090 & 3.090 & 3.090 & 3.090 \\
\hline & & & $P_{3}$ & 0.843 & 0.665 & 0.393 & 0.122 & 0.006 \\
\hline & & & $P_{r, 3}$ & 0.242 & 0.545 & 0.382 & 0.122 & 0.006 \\
\hline & & & PPV & 0.980 & 0.991 & 0.987 & 0.961 & 0.545 \\
\hline & JA & Two-stage & $C_{j, 2}$ & 4.244 & 4.877 & 5.177 & 5.306 & 5.327 \\
\hline & & & $P_{j, 2}$ & 0.030 & 0.342 & 0.705 & 0.841 & 0.852 \\
\hline & & & PPV & 0.752 & 0.972 & 0.986 & 0.988 & 0.988 \\
\hline & & Three-stage & $C_{j, 3}$ & 4.927 & 5.186 & 5.204 & 5.092 & 4.815 \\
\hline & & & $P_{j, 3}$ & 0.270 & 0.696 & 0.674 & 0.437 & 0.122 \\
\hline & & & PPV & 0.964 & 0.986 & 0.985 & 0.978 & 0.924 \\
\hline
\end{tabular}


Table 1 continued

\begin{tabular}{|c|c|c|c|c|c|c|c|c|}
\hline \multirow[t]{2}{*}{ OR } & \multirow[t]{2}{*}{ Method } & \multirow[t]{2}{*}{ Design } & \multirow[t]{2}{*}{ Indicator } & \multicolumn{5}{|l|}{$\pi_{s, 1}$} \\
\hline & & & & 0.1 & 0.3 & 0.5 & 0.7 & 0.9 \\
\hline \multirow[t]{26}{*}{1.7} & \multirow{17}{*}{ RBA } & \multirow[t]{3}{*}{ One-stage } & $\mathrm{C}$ & 5.327 & 5.327 & 5.327 & 5.327 & 5.327 \\
\hline & & & $P_{1}$ & 0.999 & 0.999 & 0.999 & 0.999 & 0.999 \\
\hline & & & PPV & 0.990 & 0.990 & 0.990 & 0.990 & 0.990 \\
\hline & & \multirow[t]{6}{*}{ Two-stage } & $C_{1}$ & 3.891 & 3.891 & 3.891 & 3.891 & 3.891 \\
\hline & & & $P_{1}$ & 0.107 & 0.758 & 0.979 & 0.999 & 1.000 \\
\hline & & & $C_{2}$ & 3.090 & 3.090 & 3.090 & 3.090 & 3.090 \\
\hline & & & $P_{2}$ & 1.000 & 1.000 & 0.998 & 0.933 & 0.330 \\
\hline & & & $P_{r, 2}$ & 0.107 & 0.758 & 0.977 & 0.932 & 0.330 \\
\hline & & & PPV & 0.915 & 0.987 & 0.990 & 0.989 & 0.971 \\
\hline & & \multirow[t]{8}{*}{ Three-stage } & $C_{1}$ & 2.576 & 2.576 & 2.576 & 2.576 & 2.576 \\
\hline & & & $P_{1}$ & 0.529 & 0.978 & 1.000 & 1.000 & 1.000 \\
\hline & & & $C_{2}$ & 2.576 & 2.576 & 2.576 & 2.576 & 2.576 \\
\hline & & & $P_{2}$ & 0.999 & 0.991 & 0.947 & 0.748 & 0.241 \\
\hline & & & $C_{3}$ & 3.090 & 3.090 & 3.090 & 3.090 & 3.090 \\
\hline & & & $P_{3}$ & 0.993 & 0.961 & 0.818 & 0.420 & 0.027 \\
\hline & & & $P_{r, 3}$ & 0.526 & 0.940 & 0.818 & 0.420 & 0.027 \\
\hline & & & PPV & 0.991 & 0.995 & 0.994 & 0.988 & 0.844 \\
\hline & \multirow[t]{6}{*}{ JA } & \multirow[t]{3}{*}{ Two-stage } & $C_{j, 2}$ & 4.244 & 4.877 & 5.177 & 5.306 & 5.327 \\
\hline & & & $P_{j, 2}$ & 0.107 & 0.758 & 0.979 & 0.998 & 0.999 \\
\hline & & & PPV & 0.915 & 0.987 & 0.990 & 0.990 & 0.990 \\
\hline & & \multirow[t]{3}{*}{ Three-stage } & $C_{j, 3}$ & 4.927 & 5.186 & 5.204 & 5.092 & 4.815 \\
\hline & & & $P_{j, 3}$ & 0.529 & 0.969 & 0.946 & 0.748 & 0.241 \\
\hline & & & PPV & 0.981 & 0.990 & 0.990 & 0.987 & 0.960 \\
\hline & \multirow{3}{*}{$\begin{array}{l}\text { Number of } \\
\text { genotypings } \\
\left(\times 10^{8}\right)\end{array}$} & One-stage & & 10.00 & 10.00 & 10.00 & 10.00 & 10.00 \\
\hline & & Two-stage & & 1.001 & 3.001 & 5.001 & 7.000 & 9.000 \\
\hline & & Three-stage & & 1.045 & 3.035 & 5.025 & 7.015 & 9.005 \\
\hline
\end{tabular}

The number of cases $=1,000$ and the number of controls $=1,000 . \pi_{s, 2}$ of the two-stage design $=1-\pi_{s, 1}$, and $\pi_{s, 2}$ of the three-stage design $=\pi_{s, 3}=\left(1-\pi_{s, 1}\right) / 2$. The number of alleles at the first stage is 500,000 in both designs. The number of alleles at the second stage of the three-stage design is 5,000 . The number of alleles at the final stage is 50 in both designs

$O R$ odds ratio, $R B A$ replication-based analysis, $J A$ joint analysis, $P P V$ positive predictive value

can be defined as selecting a target with fewer sample sizes and lower genotyping costs and maintaining the statistical powers as high as possible. Researchers need to plan casecontrol association studies based on prediction of the relationship among sample size, genotyping costs, and powers. Previous case-control association studies (Satagopan et al. 2002; Thomas et al. 2004; Zehetmayer et al. 2005) were conducted with two-stage designs. We speculated that the factors affecting powers of multistage casecontrol studies, including studies with two-stage designs, consist of the number of overall sample size and genotyping, the proportion of samples at each stage, the number of candidate alleles examined at each stage, the overall significance level, and the magnitude of the relationship between allele frequency and disease prevalence.

Some insightful simulations have been performed using various combinations of the above parameters that provided fruitful results regarding statistical powers in twostage case-control association studies (Satagopan et al. 2002, 2004; Satagopan and Elston 2003 Thomas et al. 2004; Zehetmayer et al. 2005; Skol et al. 2006; Sato et al. 2004). In particular, we note that two-stage designs can prevent decrease in the powers of RBA, despite a smaller number of genotypings than that in one-stage designs, provided that the overall sample sizes are fixed. On the other hand, Skol et al. (2006) proposed JA in a two-stage design by introducing new statistics of the linear combination of $z_{k}$ weighted by the square root of the number of subjects. They reported that the powers of JA in two-stage designs were larger than those of RBA under specific conditions. Furthermore, Sato et al. compared the powers of two-stage designs with those of three-stage designs under the conditions of a fixed total number of 940 subjects and the number of genotypings of 100,000 SNPs using the 
Table 2 Performance indicators of the two- and three-stage designs calculated by a stage-design program assuming that the number of genotypings is fixed

\begin{tabular}{|c|c|c|c|c|c|c|c|c|}
\hline \multirow[t]{2}{*}{ OR } & \multirow[t]{2}{*}{ Method } & \multirow[t]{2}{*}{ Design } & \multirow[t]{2}{*}{ Indicator } & \multicolumn{5}{|l|}{$\pi_{s, 1}$} \\
\hline & & & & 0.1 & 0.3 & 0.5 & 0.7 & 0.9 \\
\hline \multirow[t]{23}{*}{1.3} & & One-stage & $\mathrm{C}$ & 5.327 & 5.327 & 5.327 & 5.327 & 5.327 \\
\hline & & & $P_{1}$ & 0.113 & 0.113 & 0.113 & 0.113 & 0.113 \\
\hline & & & PPV & 0.919 & 0.919 & 0.919 & 0.919 & \\
\hline & $\mathrm{RBA}$ & Two-stage & $C_{1}$ & 2.804 & 2.804 & 2.804 & 2.804 & \\
\hline & & & $P_{1}$ & 0.066 & 0.291 & 0.542 & 0.739 & \\
\hline & & & $C_{2}$ & 4.110 & 4.110 & 4.110 & 4.110 & \\
\hline & & & $P_{2}$ & 0.418 & 0.252 & 0.115 & 0.032 & \\
\hline & & & $P_{r, 2}$ & 0.028 & 0.073 & 0.062 & 0.023 & \\
\hline & & & PPV & 0.736 & 0.880 & 0.862 & 0.701 & \\
\hline & & Three-stage & $C_{1}$ & 2.576 & 2.576 & 2.576 & 2.576 & 2.576 \\
\hline & & & $P_{1}$ & 0.101 & 0.374 & 0.631 & 0.807 & 0.908 \\
\hline & & & $C_{2}$ & 2.576 & 2.576 & 2.576 & 2.576 & 2.576 \\
\hline & & & $P_{2}$ & 0.573 & 0.444 & 0.302 & 0.163 & 0.049 \\
\hline & & & $C_{3}$ & 3.090 & 3.090 & 3.090 & 3.090 & 3.090 \\
\hline & & & $P_{3}$ & 0.212 & 0.114 & 0.046 & 0.011 & 0.001 \\
\hline & & & $P_{r, 3}$ & 0.022 & 0.042 & 0.029 & 0.009 & 0.001 \\
\hline & & & PPV & 0.811 & 0.895 & 0.852 & 0.639 & 0.118 \\
\hline & JA & Two-stage & $C_{j, 2}$ & 4.900 & 5.225 & 5.314 & 5.327 & \\
\hline & & & $P_{j, 2}$ & 0.028 & 0.087 & 0.110 & 0.113 & \\
\hline & & & PPV & 0.740 & 0.896 & 0.917 & 0.919 & \\
\hline & & Three-stage & $C_{j, 3}$ & 4.927 & 5.186 & 5.204 & 5.092 & 4.815 \\
\hline & & & $P_{j, 3}$ & 0.034 & 0.082 & 0.082 & 0.055 & 0.020 \\
\hline & & & PPV & 0.774 & 0.891 & 0.892 & 0.846 & 0.664 \\
\hline \multirow[t]{23}{*}{1.5} & & One-stage & $\mathrm{C}$ & 5.327 & 5.327 & 5.327 & 5.327 & 5.327 \\
\hline & & & $P_{1}$ & 0.852 & 0.852 & 0.852 & 0.852 & 0.852 \\
\hline & & & PPV & 0.988 & 0.988 & 0.988 & 0.988 & \\
\hline & RBA & Two-stage & $C_{1}$ & 2.804 & 2.804 & 2.804 & 2.804 & \\
\hline & & & $P_{1}$ & 0.215 & 0.754 & 0.956 & 0.994 & \\
\hline & & & $C_{2}$ & 4.110 & 4.110 & 4.110 & 4.110 & \\
\hline & & & $P_{2}$ & 0.974 & 0.889 & 0.654 & 0.268 & \\
\hline & & & $P_{r, 2}$ & 0.210 & 0.671 & 0.625 & 0.267 & \\
\hline & & & PPV & 0.954 & 0.985 & 0.984 & 0.964 & \\
\hline & & Three-stage & $C_{1}$ & 2.576 & 2.576 & 2.576 & 2.576 & 2.576 \\
\hline & & & $P_{1}$ & 0.288 & 0.820 & 0.973 & 0.997 & 1.000 \\
\hline & & & $C_{2}$ & 2.576 & 2.576 & 2.576 & 2.576 & 2.576 \\
\hline & & & $P_{2}$ & 0.955 & 0.884 & 0.729 & 0.457 & 0.125 \\
\hline & & & $C_{3}$ & 3.090 & 3.090 & 3.090 & 3.090 & 3.090 \\
\hline & & & $P_{3}$ & 0.843 & 0.665 & 0.393 & 0.122 & 0.006 \\
\hline & & & $P_{r, 3}$ & 0.242 & 0.545 & 0.382 & 0.122 & 0.006 \\
\hline & & & PPV & 0.980 & 0.991 & 0.987 & 0.961 & 0.545 \\
\hline & JA & Two-stage & $C_{j, 2}$ & 4.900 & 5.225 & 5.314 & 5.327 & \\
\hline & & & $P_{j, 2}$ & 0.210 & 0.703 & 0.843 & 0.852 & \\
\hline & & & PPV & 0.955 & 0.986 & 0.988 & 0.988 & \\
\hline & & Three-stage & $C_{j, 3}$ & 4.927 & 5.186 & 5.204 & 5.092 & 4.815 \\
\hline & & & $P_{j, 3}$ & 0.270 & 0.696 & 0.674 & 0.437 & 0.122 \\
\hline & & & PPV & 0.964 & 0.986 & 0.985 & 0.978 & 0.924 \\
\hline
\end{tabular}


Table 2 continued

\begin{tabular}{|c|c|c|c|c|c|c|c|c|}
\hline \multirow[t]{2}{*}{ OR } & \multirow[t]{2}{*}{ Method } & \multirow[t]{2}{*}{ Design } & \multirow[t]{2}{*}{ Indicator } & \multicolumn{5}{|l|}{$\pi_{s, 1}$} \\
\hline & & & & 0.1 & 0.3 & 0.5 & 0.7 & 0.9 \\
\hline \multirow[t]{26}{*}{1.7} & \multirow{17}{*}{ RBA } & \multirow[t]{3}{*}{ One-stage } & $\mathrm{C}$ & 5.327 & 5.327 & 5.327 & 5.327 & 5.327 \\
\hline & & & $P_{1}$ & 0.999 & 0.999 & 0.999 & 0.999 & \multirow[t]{8}{*}{0.999} \\
\hline & & & PPV & 0.990 & 0.990 & 0.990 & 0.990 & \\
\hline & & \multirow[t]{6}{*}{ Two-stage } & $C_{1}$ & 2.804 & 2.804 & 2.804 & 2.804 & \\
\hline & & & $P_{1}$ & 0.439 & 0.963 & 0.999 & 1.000 & \\
\hline & & & $C_{2}$ & 4.110 & 4.110 & 4.110 & 4.110 & \\
\hline & & & $P_{2}$ & 1.000 & 0.998 & 0.965 & 0.684 & \\
\hline & & & $P_{r, 2}$ & 0.439 & 0.961 & 0.964 & 0.684 & \\
\hline & & & PPV & 0.978 & 0.990 & 0.990 & 0.986 & \\
\hline & & \multirow[t]{8}{*}{ Three-stage } & $C_{1}$ & 2.576 & 2.576 & 2.576 & 2.576 & 2.576 \\
\hline & & & $P_{1}$ & 0.529 & 0.978 & 1.000 & 1.000 & 1.000 \\
\hline & & & $C_{2}$ & 2.576 & 2.576 & 2.576 & 2.576 & 2.576 \\
\hline & & & $P_{2}$ & 0.999 & 0.991 & 0.947 & 0.748 & 0.241 \\
\hline & & & $C_{3}$ & 3.090 & 3.090 & 3.090 & 3.090 & 3.090 \\
\hline & & & $P_{3}$ & 0.993 & 0.961 & 0.818 & 0.420 & 0.027 \\
\hline & & & $P_{r, 3}$ & 0.526 & 0.940 & 0.818 & 0.420 & 0.027 \\
\hline & & & PPV & 0.991 & 0.995 & 0.994 & 0.988 & 0.844 \\
\hline & \multirow[t]{6}{*}{ JA } & \multirow[t]{3}{*}{ Two-stage } & $C_{j, 2}$ & 4.900 & 5.225 & 5.314 & 5.327 & \\
\hline & & & $P_{j, 2}$ & 0.439 & 0.963 & 0.998 & 0.999 & \\
\hline & & & PPV & 0.978 & 0.990 & 0.990 & 0.990 & \\
\hline & & \multirow[t]{3}{*}{ Three-stage } & $C_{j, 3}$ & 4.927 & 5.186 & 5.204 & 5.092 & 4.815 \\
\hline & & & $P_{j, 3}$ & 0.529 & 0.969 & 0.946 & 0.748 & 0.241 \\
\hline & & & PPV & 0.981 & 0.990 & 0.990 & 0.987 & 0.960 \\
\hline & \multirow{3}{*}{$\begin{array}{l}\text { Number of } \\
\text { genotypings } \\
\left(\times 10^{8}\right)\end{array}$} & \multicolumn{2}{|l|}{ One-stage } & 10.00 & 10.00 & 10.00 & 10.00 & 10.00 \\
\hline & & \multicolumn{2}{|l|}{ Two-stage } & 1.045 & 3.035 & 5.025 & 7.015 & 9.005 \\
\hline & & \multicolumn{2}{|l|}{ Three-stage } & 1.045 & 3.035 & 5.025 & 7.015 & 9.005 \\
\hline
\end{tabular}

The number of cases $=1,000$ and the number of controls $=1,000 . \pi_{s, 2}$ of the two-stage design $=1-\pi_{s, 1}$, and $\pi_{s, 2}$ of the three-stage design and $\pi_{s, 3}=\left(1-\pi_{s, 1}\right) / 2$. The number of alleles at the first stage is 500,000 in both designs. The number of alleles at the second stage of the three-stage design is 5,000. The number of alleles at the final stage of three-stage design is 50, and the number of alleles at the final stage of the two-stage design is 2,525 , which provided for the number of genotypings to become equal between both designs

$O R$ odds ratio, $R B A$ replication-based analysis, $J A$ joint analysis, $P P V$ positive predictive value

Monte Carlo simulation method. They reported that the detection rate of alleles significantly affecting disease prevalence in the three-stage design was a little higher than that of the two-stage design. However, they did not generally present the sample size properties in the conditions that the detection rate of the three-stage design is higher than that of the two-stage design.

Furthermore, except for the above studies, there are few reports of more detailed evaluations concerning the performance of RBA and JA in a multistage design, though they are useful statistical methods for SNP data analyses. We thought the $n$-stage designs should have some practical advantages in maintaining the powers and reducing the number of genotypings, such as two-stage designs have more features than one-stage designs. Figure 2 shows that the three-stage design with the power of 0.70 at $\pi_{s, 1}=0.3$ can reduce the number of genotypings by 0.7 and 0.4 compared with the one-stage design with the power of 0.85 and the two-stage design with the power of 0.71 at $\pi_{s, 1}=$ 0.5 . These advantages of RBA and JA in three-stage designs may yield cost reduction of SNP genotyping for many practical studies to detect disease-related genes. Actually, at least two practical SNP-based association studies have adopted three-stage designs and succeeded in identifying disease susceptibility genes (Prentice and Qi 2006; Kathiresan et al. 2007). According to the study of Prentice et al., the power of the one-stage design was estimated to be 0.92 and that of the adequate three-stage design reached 0.75 , with the expected large reduction rate of " 0.98 " in genotyping costs compared with the one-stage design. Furthermore, Kathiresan et al. also used three-stage strategy for their GWA studies to minimize genotyping 
costs, and they estimated that the statistical power was 0.89 as maximum to detect a quantitative trait locus.

In our study, we investigated the powers and PPVs of RBA and JA by comparisons of one-, two- and three-stage designs and showed that three-stage designs are superior to two-stage designs in powers and PPVs under the condition of $\pi_{s, 1} \leq 0.5$, especially by using JA. These findings can be explained as follows.

The value of $\pi_{m, 1}$ in a two-stage design is 0.0001 and that in a three-stage design is 0.01 , and the number of alleles remaining in the final stage in the two-stage design and that in the three-stage design might therefore be almost equal. Therefore, from Table $1, P_{1}$ by the three-stage design is always larger than that by the two-stage design, and the power at the final stage (hereafter designated as $\left.P_{\text {final }}\right)$ by the two-stage design is always larger than that by the three-stage design. In addition, with increasing $\pi_{s, 1}, P_{1}$ increases and $P_{\text {final }}$ decreases. Since $P_{r, n}$ is the product of $P_{1}$ and $P_{\text {final }}$, a difference between $P_{r, 2}$ and $P_{r, 3}$ is affected from a difference between $P_{1}$ and $P_{\text {final }}$. A comparison of the ratios of $P_{1}$ by the three-stage design with those by the two-stage design $(21.1,7.4,3.9,2.5$, and 1.8) and a comparison of the ratios of $P_{\text {final }}$ by the two-stage design with those by the three-stage design $(3.7,5.6,9.4,18.4$, and 50.2 ) at each $\pi_{s, 1}$ shows that the former ratios are larger in the range of smaller $\pi_{s, 1}$ and that the latter ratios are larger in the range of larger $\pi_{s, 1}$.

Therefore, in RBA, since the influence of $P_{1}$ is larger in the range of smaller $\pi_{s, 1}$, the power by the three-stage design is larger than that by the two-stage design in that range, and since the influence of $P_{\text {final }}$ is larger in the range of larger $\pi_{s, 1}$, the power by the two-stage design is larger than that by the three-stage design in that range. Furthermore, in JA, $C_{j, 3}$ is larger than $C_{j, 2}$ in the range of 0.1-0.3 of $\pi_{s, 1}$, and $C_{j, 2}$ is larger than $C_{j, 3}$ in the range above 0.5 of $\pi_{s, 1}$. Therefore, in JA, the power by the three-stage design is larger than that by the two-stage design in the range of $0.1-0.3$ of $\pi_{s, 1}$ and the power by the two-stage design is larger than that by the three-stage design in the range above 0.5 of $\pi_{s, 1}$.

According to our simulations, $\pi_{s, 1}$, as one of the most important parameters for multistage designs, should be evaluated carefully. In the case of $\pi_{s, 1} \leq 0.5$, the threestage design by JA might be more effective than the onestage and two-stage designs. On the other hand, in the case of $\pi_{s, 1} \geq 0.6$, since the power and PPV of the two-stage design are always higher than those of the three-stage design, the three-stage design should not be used.

In this study, extensive simulations and configurations under each number of study stages showed that a multistage association study including a three-stage design is more cost effective than conventional case-control designs without sacrificing power. Researchers should therefore take account of multistage association studies, including a three-stage design corresponding to study conditions such as the total numbers of samples, alleles, and genotypings. It may be useful to conduct a prior simulation under various conditions by our program for a multistage design at the planning stage of a genome-wide association study.

\section{References}

Cohen JC (2006) Genetic approaches to coronary heart disease. J Am Coll Cardiol 48:10-14

Hirschhorn JN, Daly MJ (2005) Genome-wide association studies for common diseases and complex traits. Nature Rev 6:95-108

Kathiresan S, Manning AK, Demissie S, D'Agostino RB, Surti A, Guiducci C, Gianniny L, Burtt NP, Melander O, Orho-Melander M, Arnett DK, Peloso GM, Oardovas JM, Cupples LA (2007) A genome-wide association study for blood lipid phenotypes in the Framingham heart study. BMC Med Genet 8:1-10. http://www.biomedcetral.com/1471-2350/8/S1/S17

Moore JH, Ritchie MD (2004) The challenges of whole-genome approaches to common disease. JAMA 291:1642-1643

Prentice RL, Qi L (2006) Aspects of the design and analysis of highdimensional SNP studies for disease risk estimation. Biostatistics 7:339-354

Samani N J, Erdmann J, Hall A S, Hengsternberg C, Mangino M, Mayer B, Dixon R J, Meitinger T, Braund P, Wichmann H E, Barrett JH, König I R, Stevens S E, Szymczak S, Tregouet D A, Iles M M, Pahlke F, Pollard H, Lieb W, Cambien F, Fischer M, Ouwehand W, Path F R, Blankenberg S, Baimforth A J, Baessier A, Ball S G, Strom T M, Brænne I, Gieger C, Deloukas P, Tobin M D, Ziegler A, Thompson J R, Schunkert H (2007) Genomewide association analysis of coronary artery disease. N Engl J Med 357(5):443-453. http://www.NejM.org

Satagopan JM, Verbel DA, Venkatraman ES, Offit KE, Begg CB (2002) Two-stage designs for gene-disease association studies. Biometrics 58:163-170

Satagopan JM, Elston RC (2003) Optimal two-stage genotyping in population-based association studies. Genet Epidemiol 25:149157

Satagopan JM, Venkatraman ES, Begg CB (2004) Two-stage designs for gene-disease association studies with sample size constraints. Biometrics 60:589-597

Sato Y, Suganami H, Hamada C, Yoshimura I, Yoshida T, Yoshimura K (2004) Designing a multistage, SNP-based, genome screen for common diseases. J Hum Genet 49:669-676

Service SK, Sandkuijl LA, Freimer NB (2003) Cost-effective design for linkage disequilibrium mapping of complex traits. Am J Hum Genet 72:1213-1220

Skol AD, Scott LJ, Abecasis GR, Boehnke M (2006) Joint analysis is more efficient than replication-based analysis for two-stage genome-wide association studies. Nat Genet 30:209-213

Skol AD, Scott LJ, Abecasis GR, Boehnke M (2007) Optimal designs for two-stage genome-wide association studies. Genet Epidemiol. doi:10.1002/gepi

Tamiya G, Shinya M, Imanishi T, Ikuta T, Makino S, Okamoto K, Furugaki K, Matsumoto T, Mano S, Ando S, Nozaki Y, Yukawa W, Nakashige R, Yamaguchi D, Ishibashi H, Yonekura M, Nakami Y, Takayama S, Endo T, Saruwatari T, Yagura M, Yoshikawa Y, Fujimoto K, Oka A, Chiku S, Linsen SE, Giphart MJ, Kulski JK, Fukazawa T, Hashimoto H, Kimura M, Hoshina Y, Suzuki Y, Hotta T, Mochida J, Minezaki T, Komai K, Shiozawa S, Taniguchi A, Yamanaka H, Kamatani N, Gojobori 
T, Bahram S, Inoko H (2005) Whole genome association study of rheumatoid arthritis using 27039 microsatellites. Hum Mol Genet 14:2305-2321

The Wellcome Trust Case Control Consortium (2007) Genome-wide association study of 14,000 cases of seven common diseases and 3,000 shared controls. Nature 447:661-683
Thomas D, Xie R, Gebregziabher M (2004) Two-stage sampling designs for gene association studies. Genet Epidemiol 27:401414

Zehetmayer S, Bauer P, Posch M (2005) Two-stage designs for experiments with a large number of hypotheses. Bioinformatics 21:3771-3777 